\title{
Efficient Parallelization of Spatial Approximation Trees
}

\author{
Mauricio Marín ${ }^{1}$ and Nora Reyes ${ }^{2}$ \\ ${ }^{1}$ Computer Cs. Department, University of Magallanes, Chile \\ ${ }^{2}$ Computer Cs. Department, University of San Luis, Argentina \\ Mauricio.Marin@umag.cl
}

\begin{abstract}
This paper describes the parallelization of the Spatial Approximation Tree. This data structure has been shown to be an efficient index structure for solving range queries in high-dimensional metric space databases. We propose a method for load balancing the work performed by the processors. The method is self-tuning and is able to dynamically follow changes in the work-load generated by user queries. Empirical results with different databases show efficient performance in practice. The algorithmic design is based on the use of the bulk-synchronous model of parallel computing.
\end{abstract}

\section{Introduction}

The Spatial Approximation Tree (SAT) is a recent data structure devised to support efficient search in high-dimensional metric spaces [5, 6]. It has been compared successfully against other data structures devised for the same purpose [2,3] and update operations have been included in the original design [1, 7].

The typical query for this data structure is the range query which consists on retrieving all objects within a certain distance from a given query object. From this operation one can construct other ones such as the nearest neighbors. The distance between two database objects in a high-dimensional space can be very expensive to compute and in many cases it is certainly the relevant performance metric to optimize; even over the cost secondary memory operations [1]. For large and complex databases it then becomes crucial to reduce the number of distance calculations in order to achieve reasonable running times. The SAT is able to achieve that goal but still range query operations can be very time consuming. This makes a case for the use of parallelism.

In this paper we propose efficient parallel algorithms for range query operations upon the SAT data structure. The model of parallel computing is the so-called BSP model [10] which provides independence of the computer architecture and has been shown to be efficient in applications such as text databases and others [4, 8. The proposed algorithms can be implemented using any modern communication library such as PVM, MPI or special purpose libraries such as BSPlib or BSPpub. 
The main contribution of this paper is a low-cost method for load balancing the number of distance calculations performed by the processors. It works with any strategy of tree node distribution onto the processors. The method is selftuning and is able to adapt itself to the evolution of the work-load generated by the stream of queries being submitted to the SAT (we assume a high-traffic client-server setting in which the SAT is used as an index structure to efficiently solve user's range queries). Experimental results with different databases show that the method is able to achieve efficient performance.

\section{SAT \& BSP}

The SAT construction starts by selecting at random an element $a$ from the database $S$. This element is set to be root of the tree. Then a suitable set $N(a)$ of neighbors of $a$ is defined to be the children of $a$. The elements of $N(a)$ are the ones that are closer to $a$ than any other neighbor. The construction of $N(a)$ begins with the initial node $a$ and its "bag" holding all the rest of S. We first sort the bag by distance to $a$. Then, we start adding nodes to N(a) (which is initially empty). Each time we consider a new node $b$, we check whether it is closer to some element of $N(a)$ than to $a$ itself. If that is not the case, we add $\mathrm{b}$ to $\mathrm{N}(\mathrm{a})$. We now must decide in which neighbor's bag we put the rest of the nodes. We put each node not in $a \cup N(a)$ in the bag of its closest element of $N(a)$. The process continues recursively with all elements in $N(a)$.

The resulting structure is a tree that can be searched for any $q \in S$ by spatial approximation for nearest neighbor queries. Some comparisons are saved at search time by storing at each node a its covering radius, i.e., the maximum distance $R(a)$ between $a$ and any element in the subtree rooted by $a$.

Range queries $q$ with radius $r$ are processed as follows. We first determine the closest neighbor $c$ of $q$ among $\{a\} \cup N(a)$. We then enter into all neighbors $b \in N(a)$ such that $d(q, b) \leq d(q, c)+2 r$. This is because the result elements $q^{*}$ sought can differ from $q$ by at most $r$ at any distance evaluation, so it could have been inserted inside any of those $b$ nodes. In the process, we report all the nodes $q^{*}$ we found close enough to $q$. Finally, the covering radius $R(a)$ is used to further prune the search, by not entering into subtrees such that $d(q, a)>R(a)+r$, since they cannot contain useful elements.

In the bulk-synchronous parallel (BSP) model of computing [10,8, any parallel computer (e.g., PC cluster, shared or distributed memory multiprocessors) is seen as composed of a set of $P$ processor-local-memory components which communicate with each other through messages. The computation is organized as a sequence of supersteps. During a superstep, the processors may perform sequential computations on local data and/or send messages to other processors. The messages are available for processing at their destinations by the next superstep, and each superstep is ended with the barrier synchronization of the processors.

We assume a server operating upon a set of $P$ machines, each containing its own memory. Clients request services to a broker machine, which in turn 
distribute those requests evenly onto the $P$ machines implementing the server. Requests are queries that must be solved with the data stored on the $P$ machines. We assume that under a situation of heavy traffic the server start the processing of a batch of $Q$ queries in every superstep.

Every processor has to deal with two kind of messages, those from newly arriving queries coming from the broker, in which case a range search is started in the processor, and those from queries located in other processors that decided to continue their range search in a subtree of this processor (in this case the query is sent packed into a message, thus an initial query can give place to a number of additional query messages).

In the following discussion the main metric used to measure performance is the load balance of the computations effected by the processors. This is defined by the ratio $A / B$ where $A$ is the average of a given measure across processors, and $B$ is the maximum of the measure in any processor. The average $A / B$ is taken over all the supersteps. This is called efficiency $E_{f}$ and the value $E_{f}=1$ indicates the optimal. Speed-up is defined by $P E_{f}$. We measure computation by considering the number of distance calculations among objects during query operations and communication is the number of query messages among processors.

In the experiments below we use a $69 \mathrm{~K}$-words English dictionary and queries are composed by words selected uniformly at random. The distance between two objects is the edit distance, that is, the minimum number of character insertions, deletions, and replacements to make the two strings equal. We assume a demanding case in which queries come in pairs that are a range query for the same object but with two different radius (large and small) and the broker distribute them circularly among the processors. We use the values 1 (small) and 2 (large) for the dictionary database. The SAT is initialized with the $90 \%$ of the database and the remaining $10 \%$ are left as query objects (randomly selected from the whole database).

\section{$3 \quad$ Range Queries in Parallel}

A first point to emphasize is that the SAT structure contains nodes of very diverse number of children. Every child node causes a distance comparison, so it is relevant to be able to balance the number of distance comparisons performed in every processor per superstep. Thus it is desirable to map the tree nodes onto the processors by considering the number of distance comparisons that can be potentially performed in every sub-tree rooted at the children of the SAT's root. That is, the sub-trees associated with nodes $b$ in $N(a)$ where $a$ is root and $N(a)$ is the set defined in the previous section.

We count the total number of nodes $C(b)$ in each sub-tree with $b \in N(a)$. We then sort the $C(b)$ values and collapse these values onto the $P$ processors. To this end, we define an array $U$ of size $P$ to sum up the $C(b)$ values. For every $b \in N(a)$ such that $b$ is in decreasing order of $C(b)$ values, the node $b$ goes to processor $i$ such that $U[i]$ has the minimum sum among the $P$ elements of $U$. 
We can improve efficiency by setting upper limits $V$ to the number of distance comparisons that are performed per processor in every superstep. During a superstep, every time any processor detects that it has effected more than $V$ distance comparisons, it suspends query processing and waits until the next superstep to continue with this task. "Suspending" in our BSP realization means that all queries going down recursively in the tree in every processor $k$ are sent to processor $k$ as a message exactly as if they found out that the search has to continue in another processor (no communication cost is involved for these extra messages). Also the processors stop extracting new queries from their messages input queues. Figure 1] a shows the effect of this strategy in load balance; average efficiency improves to $E_{f}=0.95$ in this case and communication is less than $0.1 \%$.

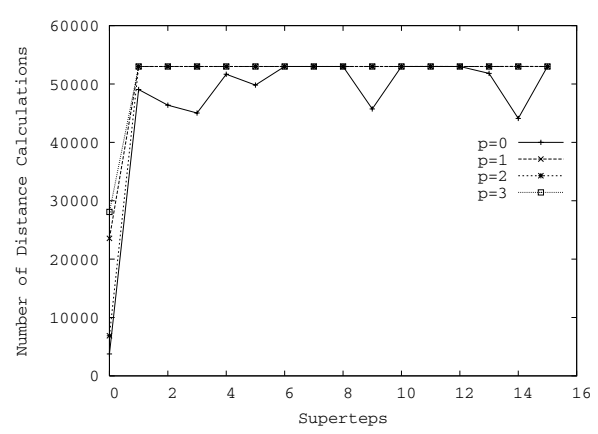

(a)

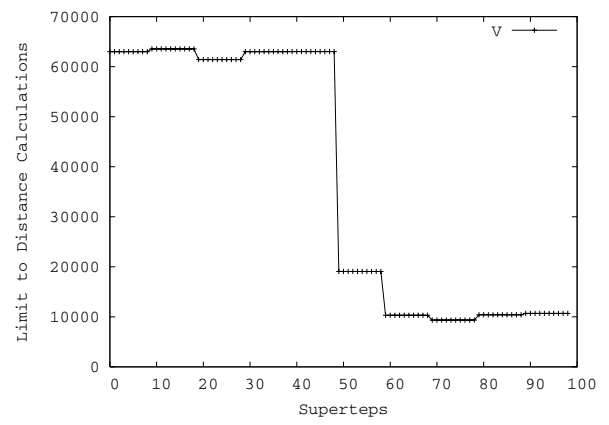

(b)

Fig. 1. (a) SAT distributed onto the processors. Number of distance calculations (comparisons) per processor versus supersteps. Demanding case for queries: Circular distribution and every object is queried with two radius. (b) Automatic and adaptive calculation of limits $V$. Halfway the work-load changes suddenly to queries with one small radius

The value $V$ should be able to adapt itself to follow changes in the workload produced by the flow of queries arriving constantly to the processors. We propose a simple and low-cost solution to this problem. The key idea is to periodically, e.g., every $s$ supersteps, collect statistics that are used to define the value of $V$ for the next sequence of $s$ supersteps. The key is to make these statistics independent of the current value of $V$ and thereby of the $s$ supersteps used to calculate them. Because of the limits $V$, supersteps can be truncated before processing all the available queries. Therefore real supersteps are not a reliable measure of the real average number of supersteps required to complete a query.

However, for each query we can know exactly when it is necessary to cross over to another processor. We can equip every query $q$ with a counter of "virtual" supersteps q.w. We distinguish these virtual supersteps from the real supersteps being executed by the BSP computer. We also keep counters of virtual supersteps $S_{v}(k)$ in each processor $k$. Every time a new query $q$ is initiated in a processor 
$k$ we set $q . w$ to be equal the batch number at which it belongs to. The broker (or the processors themselves) can assign to every query the batch number in $q . w$ before sending it to one of the processors. In a situation with $V=\infty$ the reception of every new batch of queries marks the beginning of a new superstep (in this case, virtual and real supersteps are the same). Thus every time a new query is received we set $S_{v}(k)=\max \left(S_{v}(k), q \cdot w\right)$.

We further refine the $S_{v}(k)$ values by considering that every time a query $q$ has to migrate to another processor we must set $q \cdot w=q \cdot w+1$ because it takes one virtual superstep to get there. Thus every time one of such queries $q$ arrives to a processor $k$ we also set $S_{v}(k)=\max \left(S_{v}(k), q \cdot w\right)$, and from this point onwards this query $q$ takes the value $q \cdot w=S_{v}(k)$. This ensures that queries traveling through several processors will account for the necessary (minimal) number of virtual supersteps to get their target processors and they will reflect this fact in the counters $S_{v}(k)$, a fact that will also be reflected in all other queries visiting the processor $k$.

In addition we keep counters $D(k)$ that maintain the total number of distance calculations that has been performed in every processor $k$. Thus after the processing of the $s$ real supersteps has been completed, the total number of virtual supersteps $u$ that has been completed is given by the maximum among the $P$ values $S_{v}(k)$, i.e., $u=\max 0 \leq k \leq P-1\left\{S_{v}(k)\right\}$. Thus the limit $V$ set in every processor $k$ for the next $s$ real supersteps is given by $V=d / u$ with $d=\operatorname{avg}{ }_{0 \leq k \leq P-1}\left\{D_{v}(k)\right\}$. Figure 1, b shows the effectiveness of this method.

Figures 2 a and 2. b show further experiments. In the first figure it is seen the positive effects of limits $V$ for the milder workloads which do not mix two radius for the same query object. In contrast, we show in the second figure results for a naive strategy of duplicating the SAT in every processor and distributing queries evenly for the same workloads. The results show that even in the case with no

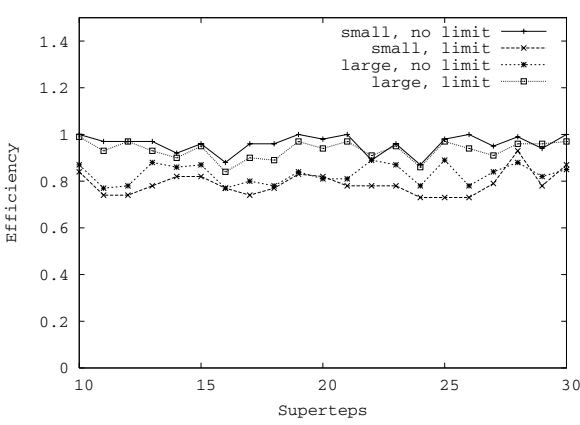

(a)

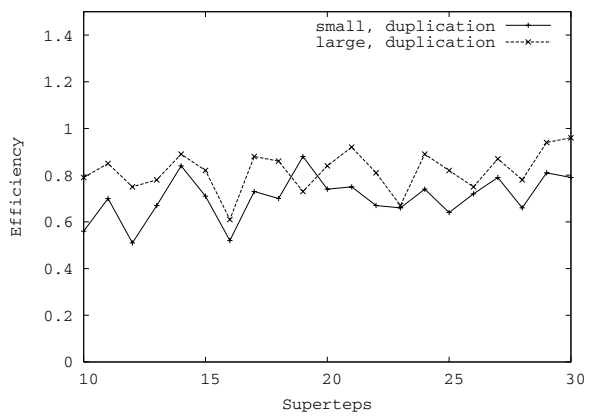

(b)

Fig. 2. (a) Efficiencies per supersteps. Proposed strategy of SAT distribution onto the processors and limits to the number of distance calculations per superstep. Two separate workloads; small and large radius. (b) Efficiencies per supersteps. Case in which a copy of the whole SAT is kept in each processor. Two separate workloads; small and large radius 
limits $V$, our proposed strategy for range queries outperform the alternative approach. Speed-ups are shown in table 1. Column "serial" is a measure of the effect of suspending queries in every superstep, i.e., the increase in supersteps (no more than 20\%).

\subsection{Other Databases and Nodes Mapping}

In figure 3 a we show results for the amount of communication demanded by an alternative mapping of SAT nodes to processors. In this case, nodes are

Table 1. Comparing different strategies

\begin{tabular}{lcccc} 
Case & \multicolumn{2}{c}{ work-load ssteps serial } & speed-up \\
\hline Duplicated & small & 432 & - & 2.85 \\
Duplicated & large & 432 & - & 3.33 \\
\hline Distributed & small & 433 & - & 3.18 \\
Distributed & large & 433 & - & 3.43 \\
\hline Dist., Limits & small & 519 & 0.17 & 3.62 \\
Dist., Limits & large & 482 & 0.10 & 3.53 \\
\hline
\end{tabular}

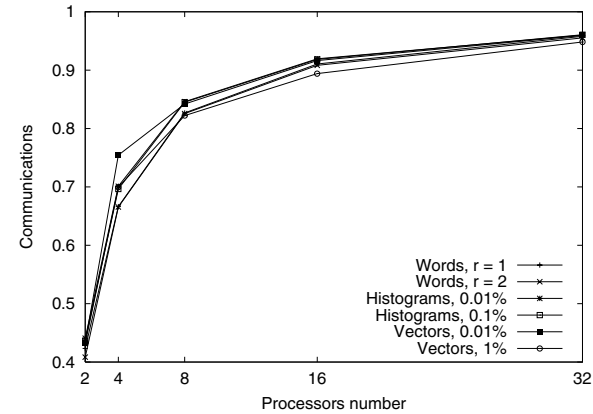

(a)

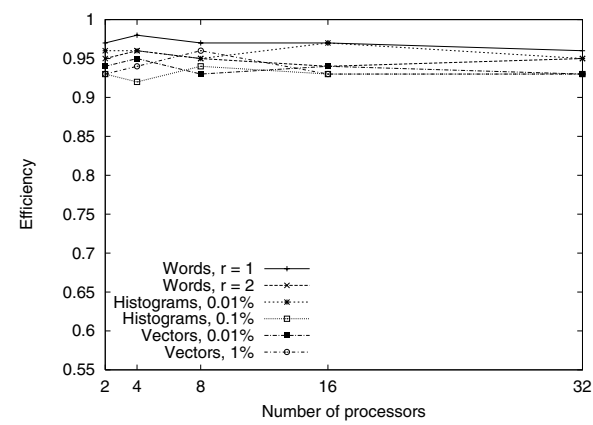

(c)

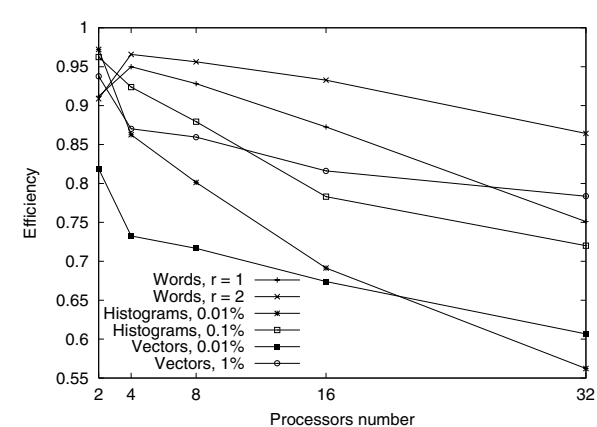

(b)

Fig. 3. (a) Effect in communication of multiplexed node distribution. (b) Efficiency achieved by the multiplexed node distribution. (c) Efficiency achieved by the multiplexed node distribution with upper limits to the number of distance calculations 
placed in a circular manner independently of their father-child-brother relations. The results were obtained with the above dictionary words and two additional databases. The new data sets are a 20-dimensional cube with points generated uniformly at random and a set of images represented by histograms defined by values of frequency amplitude. Every curve is a value for a different range query radious. Each point indicate the ration $A / B$ where $A$ is the total number of messages sent between processors and $B$ the total number of times that the function range-search was called to complete the processing of all queries. These results are in contrast with the mapping based on distance calculation suggested in this paper as the values for this case are all less than 0.1 for all databases. As expected, the amount of communication in the multiplexed approach increases significantly with the number of processors.

The figure 3 , b shows results for the efficiency $E_{f}$ with the same node distribution. Note that the vectors and histograms databases are more demanding in terms of load balance. Similar efficiency values are observed for the distribution method proposed above (selecting the least loaded processor to place a new subtree). The actual difference is in the reduced communication. Figure 3 c shows the improvement in efficiency $E_{f}$ as a result of setting upper limits $V$.

\section{Final Comments}

We have proposed parallel algorithms for range queries on a distributed SAT structure. We have focused on balancing the number of distance calculations across supersteps because this is most relevant performance metric to optimize. Distance calculation between complex objects are known to be expensive in running time. On the other hand, we have observed that the amount of communication and synchronization is indeed extremely small with respect to the cost of distance calculations. We have observed that the number of message transmissions is well below $1 \%$ with respect to the number of distance calculations. This does not consider the cost of sending solution objects as this cost has to be paid by any strategy. Also, less than 500 supersteps for processing $13 \mathrm{~K}$ queries is also a very modest amount of synchronization.

Note that we have mapped SAT nodes by considering the sub-trees belonging to the children of the root. It may happen that we have more processors than those children. This case cannot be treated by considering the mapping of subtrees one or more levels downwards the tree. Those sub-trees actually generate too few distance comparisons. We believe such case can be treated by simply using less processors than available (after all that particular SAT does not admit more parallelism) or by resorting to duplication of some sub-trees in other processors. Here we select the ones which generates more distance comparisons in an attempt to further increase parallelism by ways of dividing the query flow for that sub-trees in two or more processors. This is the subject of our future investigation.

We also tried a multiplexed approach in which every node is circularly distributed onto the processors. Efficiency is good but the cost of communication is extremely high. 


\section{Acknowledgements}

This work has been partially funded by research project Fondecyt 1040611 and RIBIDI VII.19.

\section{References}

1. D. Arroyuelo, F. Mu noz, G. Navarro, and N. Reyes. Memory-adaptative dynamic spatial approximation trees. In Proceedings of the 10th International Symposium on String Processing and Information Retrieval (SPIRE 2003), LNCS 2857, pages 360-368. Springer, 2003.

2. C. Bohm, S. Brchtold, and D. Kein. Searching in high-dimensional spaces: Index structures for improving the performance of multimedia databases. ACM Computing Surveys, 33(3):322-373, 2001.

3. V. Gaede and O. Gnnther. Multidimensional access methods. ACM Computing Surveys, 30(2):170-321, 1998.

4. M. Marín and G. Navarro. Distributed query processing using suffix arrays. In Proceedings of the 10th International Symposium on String Processing and Information Retrieval (SPIRE 2003), LNCS 2857, pages 311-325. Springer, 2003.

5. G. Navarro. Searching in metric spaces by spatial approximation. The Very Large Databases Journal (VLDBJ), 11(1):28-46, 2002.

6. G. Navarro and N. Reyes. Fully dynamic spatial approximation trees. In Proceedings of the 9th International Symposium on String Processing and Information Retrieval (SPIRE 2002), LNCS 2476, pages 254-270. Springer, 2002.

7. G. Navarro and N. Reyes. Improved deletions in dynamic spatial approximation trees. In Proc. of the XXIII International Conference of the Chilean Computer Science Society (SCCC'03), pages 13-22. IEEE CS Press, 2003.

8. D.B. Skillicorn, J.M.D. Hill, and W.F. McColl. Questions and answers about BSP. Technical Report PRG-TR-15-96, Computing Laboratory, Oxford University, 1996. Also in Journal of Scientific Programming, V.6 N.3, 1997.

9. URL. BSP PUB Library at Paderborn University, http://www.unipaderborn.de/bsp.

10. L.G. Valiant. A bridging model for parallel computation. Comm. ACM, 33:103111, Aug. 1990. 